\title{
AN EFFICIENT MODEL FOR REDUCING SOFT BLOCKING PROBABILITY IN WIRELESS CELLULAR NETWORKS
}

\author{
Edem E. Williams ${ }^{1}$ and Daniel E. Asuquo ${ }^{2}$ \\ ${ }^{\mathbf{1}}$ Department of Mathematics/Statistics and Computer Science \\ University of Calabar, PMB 1115, Calabar-Nigeria \\ ${ }^{2}$ Department of Computer Science \\ University of Uyo, PMB 1017, Uyo-Nigeria
}

\begin{abstract}
One of the research challenges in cellular networks is the design of an efficient model that can reduce call blocking probability and improve the quality of service $(Q o S)$ provided to mobile users. Blocking occurs when a new call cannot be admitted into the network due to channel unavailability caused by limited capacity or when an ongoing call cannot be continued as it moves from one base station to another due to mobility of the user. The proposed model computes the steady state probability and resource occupancy distribution, traffic distribution, intra-cell and inter-cell interferences from mobile users. Previously proposed models are reviewed through which the present model is built for use in emerging wireless networks so as to obtain improved QoS performance. The developed model is validated through simulations in MATLAB and its equations implemented using Java Programming Language. The results obtained indicate reduced call blocking probability below threshold.
\end{abstract}

\section{KEYWORDS}

Call Blocking Probability Reduction, Cellular Networks, Quality of Service \& Computer Simulations

\section{INTRODUCTION}

In cellular mobile networks, call blocking can occur as either new call blocking or handoff call blocking. The former refers to blocking of a new call request due to lack of available channel while the later refers to blocking of a call in the new cell as the mobile moves from its originating base station (BS) to a new BS. Ideally, during handoff or handover, the distributed mobile transceivers move from cell to cell during an ongoing continuous communication and switching from one cell frequency to a different cell frequency is done electronically without interruption and without a BS operator or manual switching. Typically, a new channel is automatically selected for the mobile unit on the new BS which will serve it. The mobile unit then automatically switches from the current channel to the new channel and communication continues. The two kinds of arriving calls to a cell site are shown in figure 1. Call blocking probability is one of the quality of service (QoS) parameters for performance evaluation in wireless cellular networks. According to [1], for better QoS it is desirable to reduce the call blocking probability. Call blocking is perceived negatively by users because it results to degradation in required QoS and developing an efficient model to reduce its probability in cellular networks is a growing research aimed at improving overall cellular system performance. 


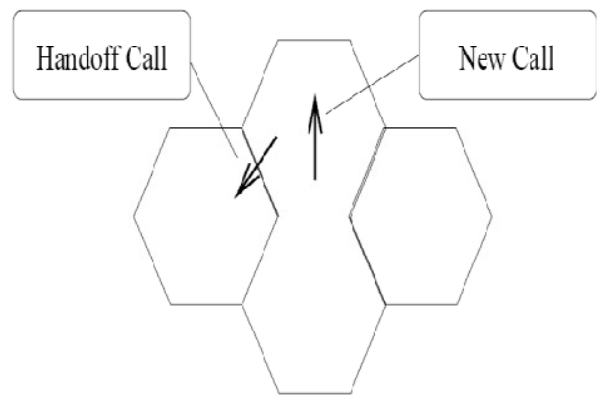

Figure 1: The two kinds of arriving calls to a cell site

The existing Visafone network suffers more of call blocking causing more calls to be rejected due to channel unavailability, as shown in figure 2. It indicates that only a few of its BSs within the region under study were able to carry traffic load beyond 400 Erlang per cell. This is definitely not a good characteristic of a reliable and efficient network. The key Performance Indicators (KPIs) usually measured by the regulatory bodies or commissions include Call Setup Success Rate (CSSR), Call Completion Rate (CCR), Standalone Dedicated Control Channel and Handover Success Rate (SDCCH), Call Data Rate (CDR) and Traffic Channel Congestion with or without Handover (TCHCon). The network operators could be rated on excellent, good, improvement, fluctuation, poor, slight decay and mostly steady below threshold on the KPIs. It is important to note that a rating of improvement does not mean the target key performance indicator is met. Rather, it means that the trend to reach the threshold is progressing towards the set target of the indicator, taking into consideration the challenges the operators are facing daily. It is therefore important that the operators continue working towards meeting the set target by adopting improved architectures despite challenges faced in infrastructure, upgrade, and service delivery.

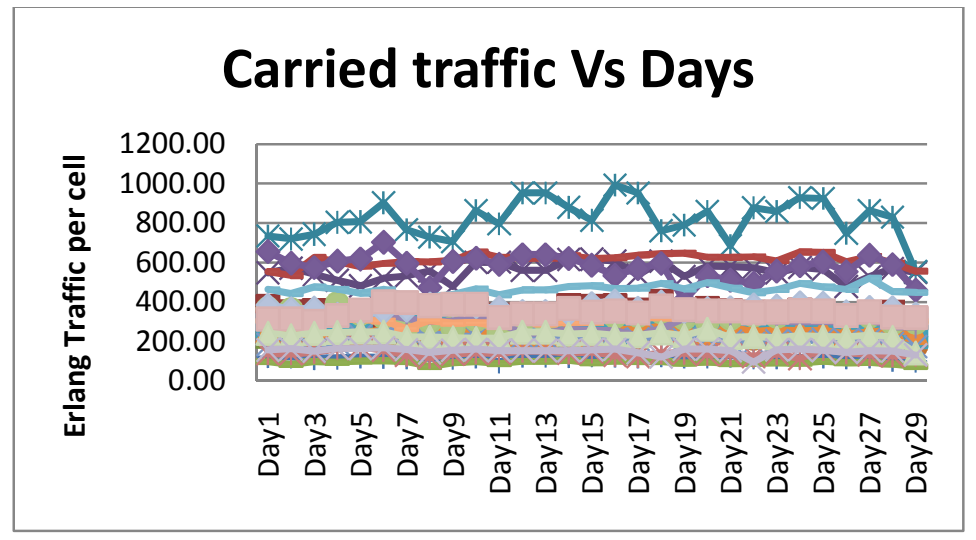

Figure 2: Visafone BS's Carried Traffic Load for 30 days

Several proposed models or techniques for reducing call blocking probability exist in literature either for reducing new call blocking [2], or reducing handoff blocking [3,4,5] or reducing both $[6,7,8]$. Whatever the case, there is a tradeoff between reducing handoff probability and new call blocking probability. A proposed method which attempts to optimally reduce handoff failures in mobile networks without significant increase in blocking probability of originating calls within a cell is highly desirable. However, call blocking model could either be said to be hard or soft. In hard call blocking models, the blocking probability is evaluated with fixed-valued parameters regardless of the channel and traffic conditions. In a Code Division Multiple Access (CDMA)based network, the interference-limited nature makes it difficult to achieve accurate admission 
control. Because of the co-channel interference, the amount of resources (power, bandwidth) required by each user is dependent on the number of users in the system, their geographical locations, and physical channel conditions. In soft blocking models, the blocking probability is evaluated taking note of interference nature of the CDMA network. This work develops and implements a soft blocking probability reducing model for new call request in the uplink of a CDMA cellular network. A well established CDMA network named Visafone is studied in the South-South Zone of Nigeria's Niger Delta region.

The rest of this paper is organized as follows: section 2 reviews existing literature on call blocking probability estimation for both hard and soft blocking while section 3 presents the system design for the proposed model for reducing soft blocking probability with the developed algorithm. In section 4, the pseudo code for the developed algorithm is presented, the model's accuracy is validated through computer simulations in MATLAB and the equations implemented in Java. Section 5 presents the simulation results and conclusion giving direction for future works.

\section{LITERATURE REVIEW}

In a wireless network composed of several BSs serving some mobile users, user's power is limited to some given maximal value. The same frequency spectrum is available to all BSs (i.e., the frequency reuse factor is unity, 1). For a CDMA network, the interference of single user detection is regarded as noise. MSs and BSs are both assumed to be uniformly and randomly distributed on an infinite plane, but with different terminal densities (coverage areas). Blocking occurs in a network when due to limited capacity at least one link on the route is not able to admit a new call. Thus, such a user will not be able to subscribe to a particular channel. The following section discusses the two types of call blocking for CDMA BSs.

\subsection{Hard Call Blocking}

Hard blocking occurs when arriving calls to a network are blocked due to lack of available channels irrespective of the traffic characteristic and channel conditions [2, 5].

\subsubsection{Erlang-B Model}

The Erlang-B formula in equation (1) is used to compute hard blocking as a function of the number of available channels and the offered load. The equation is based on analytical probability theory and can be used when the following assumptions are satisfied.

i. $\quad$ All call attempts are Poisson distributed with exponential service time

ii. Blocked calls are cleared (BCC) in the system and that the caller tries again later

$P_{B}=\frac{A^{N} / N !}{\sum_{i=0}^{N}\left(A^{i} / i !\right)}$

In equation (2.3), $P_{B}=$ probability of blocking,

$A=$ offered traffic in Erlang,

$N=$ number of channels in the cell, and

$\mathrm{i}=$ number of busy channels

Thus, there is no queuing and no retry for unsuccessful calls with the Erlang-B model.

\subsubsection{Extended Erlang B Model}

The Extended Erlang B Model uses the same formula and assumptions as Erlang-B model except that a percentage of callers retry their calls until they are serviced. This model is commonly used 
for standalone trunk groups with a retry probability such as a MODEM pool. The model is as expressed in equation (2).

$$
P_{b}=\frac{A^{N} / N !}{\sum_{k}^{N} A^{k} / k !}
$$

Where, $\mathrm{k}$ is the number of busy channels, $\mathrm{N}$ is the number of servers (trunks), $\mathrm{A}$ is the traffic density in Erlang, and $P_{b}$ is the blocking probability.

The Extended Erlang B traffic model is used by telephone system designers to estimate the number of lines required for public switch telephone network connections (trunks) or private wire connections and takes into account the additional traffic load caused by blocked callers immediately trying to call again if their calls are blocked. It therefore, allows retry of unsuccessful calls and assumes infinite population of callers (sources). This traffic model may be used where no overflow facilities are available from the trunk group being designed.

\subsubsection{Erlang-C Model}

In the Erlang $\mathrm{C}$ Model, the system is designed around the queuing theory. The caller makes one call and is held in a queue until answered, so the formula expresses the waiting probability. Just as the Erlang B formula, Erlang $\mathrm{C}$ assumes an infinite population of sources, which jointly offer traffic of $A$ Erlangs to $N$ servers. However, if all the servers are busy when a request arrives from a source, the request is queued. An unlimited number of requests may be held in the queue in this way simultaneously.

This formula calculates the probability of queuing offered traffic, assuming that blocked calls stay in the system until they can be handled. This formula is used to determine the number of agents or customer service representatives needed to staff a call centre a specified desired probability of queuing. The blocking probability or the delay probability (waiting probability) is given in equation 3.

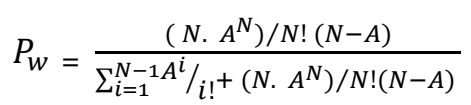

where,

$A$ is the total traffic offered in units of erlangs

$N$ is the number of servers; $i$ is the number of busy servers

$P_{W}$ is the probability that a customer has to wait for service

It is assumed that the call arrivals can be modeled by a Poisson process and that call holding times are described by a negative exponential distribution. A common use for Erlang $\mathrm{C}$ is modeling and dimensioning call center agents in a call center environment. It can also be used to determine bandwidth needs on data transmission circuits.

\subsubsection{Engset Formula}

The Engset formula is used to determine the probability of congestion occurring within a telephony circuit group. It deals with a finite population of $S$ sources rather than the infinite population of sources that Erlang assumes. The formula requires that the user knows the expected peak traffic, the number of sources (callers) and the number of circuits in the network.

Engset's formula given in equation (4) is similar to the Erlang-B formula; however one major difference is that the Erlang's equation assumes an infinite source of calls, yielding a Poisson arrival process, while Engset specifies a finite number of callers. Thus Engset's equation should 
be used when the source population is small. But for population sources greater than 200 users, extensions or customers, it becomes similar to Erlang-B model $[9,10]$.

$$
\begin{aligned}
P_{b}= & A^{N}\left(\begin{array}{l}
S \\
N
\end{array}\right) / \sum_{i=0}^{N} A^{i}\left(\begin{array}{l}
S \\
i
\end{array}\right) \\
& \text { where, } \\
& A=\text { offered traffic intensity in Erlangs, from all sources } \\
& S=\text { number of sources of traffic } \\
& N=\text { number of circuits in group } \\
& \mathrm{P} b=\text { probability of blocking or congestion }
\end{aligned}
$$

In the traditional flat cellular networks, the Erlang-B model is generally used to describe the limitation in physical resources independent of the quality experienced on the radio interface (i.e. hard blocking). With the increasing complexity of CDMA cellular networks, the required assumptions are no more valid and the Erlang-B formula is found to overestimate the capacity. Thus, to properly account for the quality of service experienced at the BS, soft blocking should be modeled and evaluated.

\subsection{Soft Call Blocking}

Soft blocking is related to the amount of interference in a network. There may be plenty of channels available at a BS but since there are many users in the same cell already, the interference level is such that adding an additional user would increase the interference above a predetermined threshold. The call is therefore denied. Modeling soft blocking due to interference is important and is considered a major aspect of this paper.

In [11], three explicit analytical models (single random trials (SRT), repeated random trials (RRT), and least busy fit (LBF)) were developed for evaluating the request blocking probability of movie files in video-on-demand $(\mathrm{VoD})$ systems under three server selection schemes. The authors reported that the choice of server selection schemes can significantly affect the blocking probability performance of the system, and validated the accuracy of the analytical models through simulation.

A rapid and accurate method for evaluating the quality of service (QoS) perceived by the users in the uplink of wireless cellular networks was proposed in [2]. In doing so, the author aimed at accounting for the dynamics induced by the arrivals and the departures of users. The evaluated QoS was in terms of the blocking probability for streaming users and the throughput for elastic calls. The blocking probability of streaming users was evaluated using the Kaufman-Roberts algorithm as in $[12,13]$, whereas the throughput of elastic calls was evaluated using a multi-class processor sharing model. The research in [14] modeled soft blocking in multi-cell CDMA systems as an independent birth and death process at each cell. The model predicts the distribution of the number of calls connected to a base station.

In [15], the authors presented an analytical model for the estimation of the blocking probability as a function of the offered traffic per user in a cellular environment, where capacity is determined by hard blocking and the average number of users per cell is small. Using statistical model, they concluded that the number of mobiles audible to a base station with the strongest signal has a Poisson distribution, which mean is given in terms of the mean densities of mobile and base stations and the parameters of the attenuation law. The model does not represent a tool for detailed network planning.

In [16], the outage probability is considered a performance measure for real-time traffic in wireless networks. They observed that the blocking and outage probabilities do not have closedform expressions as they strongly depend on the traffic characteristics (call duration, bit rate requirement, etc.), the radio conditions (fading, shadowing, noise, interference, etc.), the 
considered admission and outage policies. They assumed that the admission and outage policies satisfy a certain monotonicity property. Their results are applied to the uplink and the downlink of CDMA networks. In [17], a wireless network with beam-forming capabilities at the receiver is considered. They derived the blocking probabilities for calls in the system, under different traffic policies. For a set of co-channel transmitters, their success probabilities for being captured by separate antenna beams are computed. These success probabilities are taken into account in the queuing model of the system. Their analytical and numerical results show that adaptive beamforming at the receiver reduces the blocking probability of calls and increases the total carried traffic of the system.

In [18], a new resource-dimensioning concept based on both the allowable noise-rise and traffic statistics is presented. The soft blocking probability based on outage probability and the assumption of the Poisson arrival and exponential services time are first derived, with a consistent view on traffic dimensioning. The relationship between outage probability, soft blocking probability and hard blocking probability is discussed. The authors in [19] focused on the call blocking probabilities calculation in a WCDMA cell with fixed number of channels and finite number of traffic sources. They proposed the use of the Engset Multi-rate Loss Model (EnMLM) in the uplink direction, which incorporates local blockings. The call admission depends on the availability of the required channels. To analyze the system, they formulated an aggregate onedimensional Markov chain.

The work in [4] studied the QoS in terms of blocking and dropping probabilities, but the interference between the users was not taken into account explicitly. The work in [20] studied the QoS in wireless local area and sensor networks whereas this work focuses on wireless cellular networks. Certain models for soft blocking evaluation assumes that there is a constant number of users $N$ in the cell, power control is perfect, and each user requires the same signal bit energy to noise spectral density $E_{b} / N_{o}$. From the above review, it appears studies on soft blocking have not considered interference under imperfect power control which this work considers by extending the Kaufman-Roberts algorithm under the assumption that in reality, none of these assumptions holds. The reason is that the number of active users in a cell is Poisson distributed with mean arrival and exponential service time $\lambda / \mu$. Furthermore, due to voice activity, each user is ON with probability, $v$ and OFF with probability $(1-v)$ and each user requires a different $E_{b} / N_{o}$ to achieve a desired bit error rate or communication signal quality.

\section{PROPOSED MODEL FOR REDUCING CALL BLOCKING PROBABILITY}

In CDMA systems, signals of each MS can be modeled as interfering noise for the others, leading to degradation in service. Adopting imperfect power control in CDMA wireless networks is to regulate the transmission power levels of MS such that each user obtains a satisfactory QoS. This goal is more precisely stated as to achieve a certain SINR regardless of channel conditions while minimizing the interference and battery usage, and hence improving the overall performance. Modeling the uplink system interference involves taking into consideration the cell model, path loss model, power control, and simulation parameters. The proposed system design is shown in figure 3 and implemented in six algorithmic stages. The stages are:

(i) modeling the state of a cell

(ii) Computation of total uplink interference

(iii) Computation and comparison of maximum effective cell load with threshold

(iv) Computation of soft blocking probability when cell load threshold is exceeded

(v) Blocking or accepting new call based on computed probability

(vi) Storage of generated results and updated system parameters 


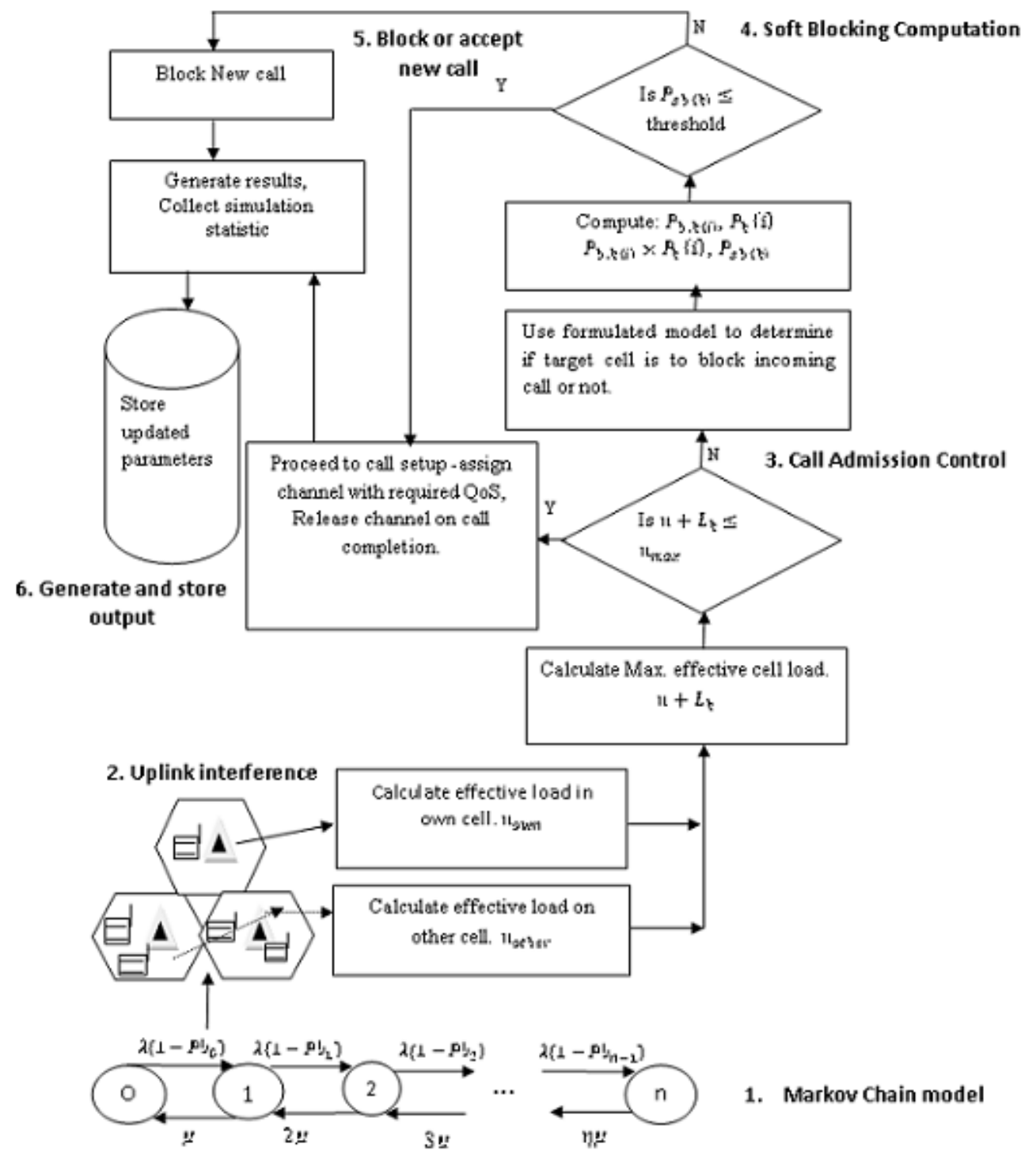

Figure 3: Proposed System Design for Soft Blocking Probability Evaluation

\section{Stage1: Modeling the State of a Cell}

Cell Model: In our model, each cell blocks newly arriving calls with a state dependent probability illustrated using Markov Chain in figure 3. The state of a cell is the number of users currently in that cell. Other cells simply contribute interference which causes blocking with some probability, $P_{b(i)}$ which is assumed to depend on the state $i$ of the current cell. We model the state of a cell as a birth and death process and showed that the arrival rate is thinned by the blocking probability, $\lambda\left(1-P_{b(i)}\right)$, while the departure rate is $i u$.

\section{Stage 2: Computation of Total Uplink Interference}

CDMA systems consider soft blocking taking note of interference from mobiles in own cell and other cells. Therefore in the uplink, the interference experienced by a certain mobile is related to the load distribution within the network. The total interference is computed considering own-cell 
interference, other-cell interference, and interference due to an empty system referred to as thermal noise i.e $I_{\text {total }}=I_{\text {own }}+I_{\text {other }}+N_{o}$. This is represented in figure 3 in the hexagonal 3cell structure. The more users are active at the BS, the larger is the multi-access interference at the BS and the higher are the transmit powers required by mobiles to fulfill their $E_{b} / N_{o}$ requirements.

SINR Calculation: The signal-to-interference-noise ratio SINR of each and every connection in the uplink depends on the power emitted by mobile users, own-cell interference $I_{\text {own }}$, other-cell interference $I_{\text {other }}$, thermal noise $N_{0}$, and multi user detection factor, $\beta$ as given in equation 5 .

$\operatorname{SINR}_{U L}=\frac{S}{(1-\beta) I_{\text {own }}+I_{\text {other }}+N_{0}}$

where the received signal, $S=P_{k}-\max \left(L-G_{t x}-G_{r x}\right)$, and $P_{k}$ is the transmit signal power, $G_{t x}$ is the transmitting antenna gain, $G_{r x}$ is the receiving antenna gain and $L$ is the path loss propagation (attenuation) model from MS-BS.

Path Loss Model: Considering a BS antenna height $\Delta h_{b}$ of 15metres and log-normally distributed shadowing $(\log \mathrm{F})$ with standard deviation of $10 \mathrm{~dB}$, the path loss is calculated based on equation (6) as in [21] as follows:

$L=40\left(1-4 \times 10^{-3} \Delta h_{b}\right) \log (d)-18 \log \left(\Delta h_{b}\right)+21 \times \log (f)+80$

$L=127.8+37.6 \log (d)+\log (F)=137.8+37.6 \log (d)$

where $d$ is the MS-BS separation in kilometers.

Users connect to the BS per cell and each mobile connecting to one BS only at any given time. The $i_{t h}$ mobile transmits with a nonnegative uplink power level of $0 \leq P_{i} \leq P_{\max } \forall_{i}$, where $P_{\max }$ is a sufficiently large upper-bound imposed for technical reasons. The received power at the $l_{t h}$ BS, $x_{i l}$, is the attenuated version of the transmitted power level, $x_{i l}=h_{i l} P_{i}$, where the quantity $h_{i l}\left(0<h_{i l}<1\right)$ represents the slow-varying channel gain (excluding any fading).

\section{Stage 3: Computation and Comparison of Maximum Effective Cell load with Threshold}

Power Model: When a call arrives to the cell, the noise rise is estimated and if it exceeds a maximum predefined threshold, the call is blocked and lost. Noise rise is the ratio of total received power at the $\mathrm{BS}, I_{\text {total }}$, to the thermal noise power, $N_{o}$ given in equation (8) as:

$N R=\frac{I_{\text {total }}}{N_{o}}=\frac{I_{\text {own }}+I_{\text {other }}+N_{O}}{N_{o}}$

The cell load $n$, is defined as the ratio of the received power from all active users to the total received power as given in equation (9). The cell load threshold must not be exceeded for call admission at any given state of the cell.

$n=\frac{I_{\text {own }}+I_{\text {other }}}{I_{\text {own }}+I_{\text {other }}+N_{o}}$

The noise rise is related to the cell load given in equation (10) as follows:

$N R=\frac{I_{\text {total }}}{N_{O}}=\frac{I_{\text {own }}+I_{\text {other }}+N_{O}}{N_{O}}=\frac{1}{\frac{N_{O}}{I_{\text {own }}+I_{\text {other }}+N_{O}}}=\frac{1}{1-\frac{I_{\text {other }}+N_{O}}{I_{\text {own }}+I_{\text {other }}+N_{O}}}=\frac{1}{1-n}$

Thus, instead of using noise rise, the cell load can be used to determine call admission. 


\section{Stage 4: Computation of Soft Blocking Probability when Cell load Threshold is Exceeded}

Simulation Model: In CDMA network, the cell load, $n$ is interpreted as shared resource and the load per cell (loading factor), $L_{k}$ as resource requirement. The application of the KaufmanRoberts algorithm postulates a discrete shared resource and discrete service requirements. Thus, in order to calculate the new call blocking probabilities of different service classes, we make discrete the loading factor and the cell load by introducing a cell load unit $g$ of which $n_{\max }$ is an integer multiple. This will help us determine the system state probabilities. Thus, the resulting capacity and resource requirements are:

$$
C=\frac{n_{\max }}{g} \quad \text { and } \quad r_{k}=\operatorname{round}\left(\frac{L_{k}}{g}\right)
$$

Since a state corresponds to the resources occupied when all users are active. We denote by $c=n_{\text {own }} / g$ as the number of occupied resources by the active users and introduce a random variable $\Lambda$ for the number of occupied resources. Still assuming no local blocking occurs, the probability $\Lambda(\mathrm{c} \mid \mathrm{i})$ that $\mathrm{c}$ resources are occupied in state $\mathrm{i}$ (bandwidth occupancy) is computed from equation (11):

$\Lambda(\mathrm{c} \mid i)=\sum_{k=1}^{K} P_{k}(i)\left[v_{k} \Lambda\left(\mathrm{c}-\mathrm{r}_{\mathrm{k}} \mid i-\mathrm{r}_{\mathrm{k}}\right)+\left(1-v_{k}\right) \Lambda\left(\mathrm{c} \mid i-\mathrm{r}_{\mathrm{k}}\right)\right]$

$$
\text { for } i=1, \ldots, i_{\text {max }} \text { and } c \leq i \text {, where } i_{\text {max }} \text { is the highest reachable system state. }
$$

The resource occupancy distribution is computed according to equation (11). So, using the theorem of total probability we derive the local blocking probability (blocking factor) as in [8] as:

$P_{b, k}(i)=\sum_{c=0}^{i} \Lambda(\mathrm{c} \mid i) P_{b, k}(c)$

Again, we denote by $P_{k}(i)$, the probability that the system is in state $i$ or the probability that state $i$ is reached by a new call of service $k$ or the probability that state $i$ is reached from state $i-r_{k}$ as follows:

$P_{k}(i)=\frac{\mathcal{P}\left(i-r_{k}\right)\left(1-P_{b, k}\left(i-r_{k}\right)\right) \alpha_{k} \frac{r_{k}}{i}}{\sum_{i=0}^{c} \mathcal{P}(i)}$

where,

$\mathcal{P}(i)=\left\{\begin{array}{cl}1, & \text { for } i=0 \\ \sum_{k=1}^{K} \mathcal{P}\left(i-r_{k}\right)\left(1-P_{b, k}\left(i-r_{k}\right)\right) \alpha_{k} \frac{r_{k}}{i} & \text { for } i=1, \ldots, C \\ 0, & \text { else }\end{array}\right.$

This probability depends on $\mathcal{P}\left(i-r_{k}\right)$ and $P_{b, k}\left(i-r_{k}\right)$ which are known for all states $c$ with $c<i . \alpha_{k}, r_{k}, \mathcal{P}(i), P_{b, k}(i)$ are the parameters of the model with infinite number of sources. Still observing the steady state probabilities given in equation (14), we obtain the total (soft) blocking probability for a service-class $k$ as the sum of all state probabilities $P_{k}(i)$ multiplied with the blocking probabilities $P_{b, k}(i)$, for all reachable states as shown in equation (15):

$P_{S b(k)}=\sum_{i=0}^{i_{\max }} P_{b, k}(i) * P_{k}(i)$ 
International Journal of Wireless \& Mobile Networks (IJWMN) Vol. 6, No. 4, August 2014

\section{Stage 5: Blocking or Accepting New Call based on Computed Probability}

The developed model is aimed at enhancing the acceptance of more user calls into the system to improve overall utilization of scare network resources. Thus, a call is blocked when the blocking probability value is greater than the predefined threshold of 0.01given in [15] or at worst 0.02 , otherwise, it is accepted, assigned a channel with needed QoS requirement and the channel released on call completion.

In [22], an intelligent CAC scheme was developed where fuzzy logic technique was adopted in the admission decision. Calls admissions were termed strongly accepted, weakly accepted, weakly rejected and strongly rejected depending on the output value of the multi-criteria parameters in the input to the call admission controller. Their results show the capability of fuzzy logic to improve system performance by accepting more user calls.

\section{Stage 6: Storage of Generated Results and Updated System Parameters}

At this stage, the result obtained from the computed soft blocking probability which is used to determine the acceptance or blocking of the new call is stored. Similarly, system parameters generated are also stored in the database so that the next state of a cell can be determined from parameters from the previous state. The objective is to improve battery live, reduce inter-cell interference, maintain desired service quality, and maximize utilization of network resource for improved system performance.

\section{MODEL IMPLEMENTATION}

Figure 4 shows the pseudo code for the developed algorithm. The model was validated through simulations in MATLAB and its equations were implemented in Java programming language. The results obtained are as shown in figures 5-7 for the three performance measures listed below. Table 1 indicates the simulation parameters.

Performance Measure $1\left(P_{s b(k)}\right.$ vs. $\alpha_{k}$ at different $R_{k}$ values): The impact of data rates on soft blocking for given offered load.

Performance Measure $2\left(P_{s b(k)}\right.$ vs. $\alpha_{k}$ at different $E_{b} / N_{o}$ values): The impact of signal energy per bit to noise spectral density for given offered load.

Performance Measure $3\left(P_{s b(k)}\right.$ vs. $\alpha_{k}$ at different $I_{\text {other }}$ values): The impact of other-cell interference on soft blocking for given offered load.

In figure 5, the developed system accepts input parameters and runs numbers of simulations for performance measure 1(i.e. model 1). The result obtained indicates that at different traffic data rates for given offered load to the system, the soft blocking probability values are higher for services that require higher data rates and vice versa. Nevertheless, the overall performance of the system is improved as the soft blocking probability values obtained are less than 0.02 showing significant reduction.

In figure 6, the system evaluates the impact of each user's signal energy per bit to noise spectral density $E_{b} / N_{o}$ on soft blocking since in reality, it value cannot be fixed for all service classes in a CDMA network. This parameter is sometimes used to ascertain the user's QoS requirement. For different values of $E_{b} / N_{o}$ input for performance measure 2 (i.e. model 2), the soft blocking probability is reduced significantly indicating that more user calls are admitted into the system for resource sharing.

In figure 7 , the impact of other-cell interference $I_{\text {other }}$ on soft blocking probability is simulated for given traffic load considering performance measure 3 (i.e. model 3). The results indicate that though the soft blocking probability values obtained are higher for higher values of $I_{\text {other }}$ 
International Journal of Wireless \& Mobile Networks (IJWMN) Vol. 6, No. 4, August 2014

indicating the negative effect of inter-cell interference on CDMA networks, the values are significantly reduced thus allowing lower blocking rates.

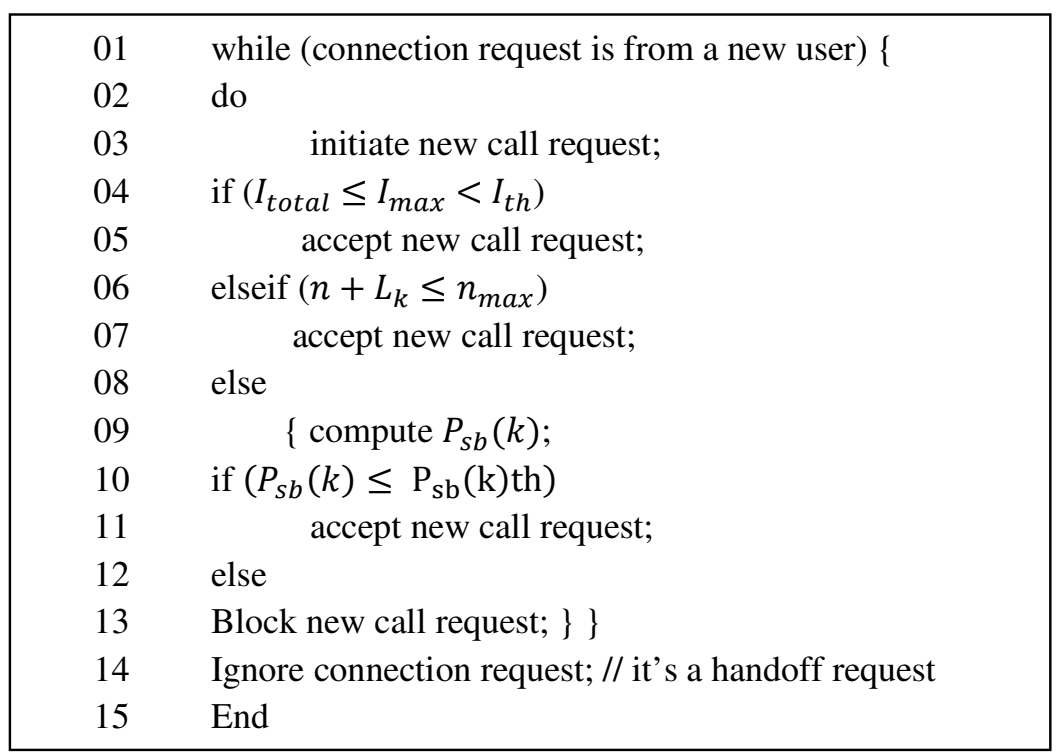

Figure 4: Pseudo code for the developed algorithm

Table 1: Simulation parameters

\begin{tabular}{|l|l|}
\hline Parameter & Value \\
\hline Chip rate, $\mathrm{W}$ & $5 \mathrm{MHz}$ \\
\hline BS thermal noise, $N_{o}$ & $-174 \mathrm{dBm}$ \\
\hline Data rate, $R_{k}$ & $144 \mathrm{Kbps}, 253 \mathrm{Kbps}, 384 \mathrm{Kbps}$ \\
\hline Voice activity factor, $v_{k}$ & $0.65,1$ \\
\hline Signal bit energy over noise spectral density, $E_{b} / N_{o}$ & $3-7 \mathrm{~dB}$ \\
\hline Multi user detection factor, $\beta$ & 0.78 \\
\hline Transmitting antenna gain, $G_{t x}$ & $11 \mathrm{dBi}$ \\
\hline BS antenna height, $\Delta h_{b}$ & $15 \mathrm{~m}$, omnidirectional \\
\hline MS transmitted power signal & $21-30 \mathrm{dBm}$ \\
\hline Maximum cell load, $n_{\max }$ & 0.8 \\
\hline Values of other-cell interference, $I_{\text {other }}$ & $5 e^{-19} \mathrm{~mW}-6 e^{-18} \mathrm{~mW}$ \\
\hline Offered load, $\alpha_{k}$ & $0.2-2.0 \mathrm{Erlang}$ \\
\hline Loading factor, $L_{k}$ & $0.05,0.1,0.15,0.2,0.25$ \\
\hline Maximum number of states, $i_{\max }$ & 4 \\
\hline Number of service class, $k$ & 3 \\
\hline
\end{tabular}


International Journal of Wireless \& Mobile Networks (IJWMN) Vol. 6, No. 4, August 2014

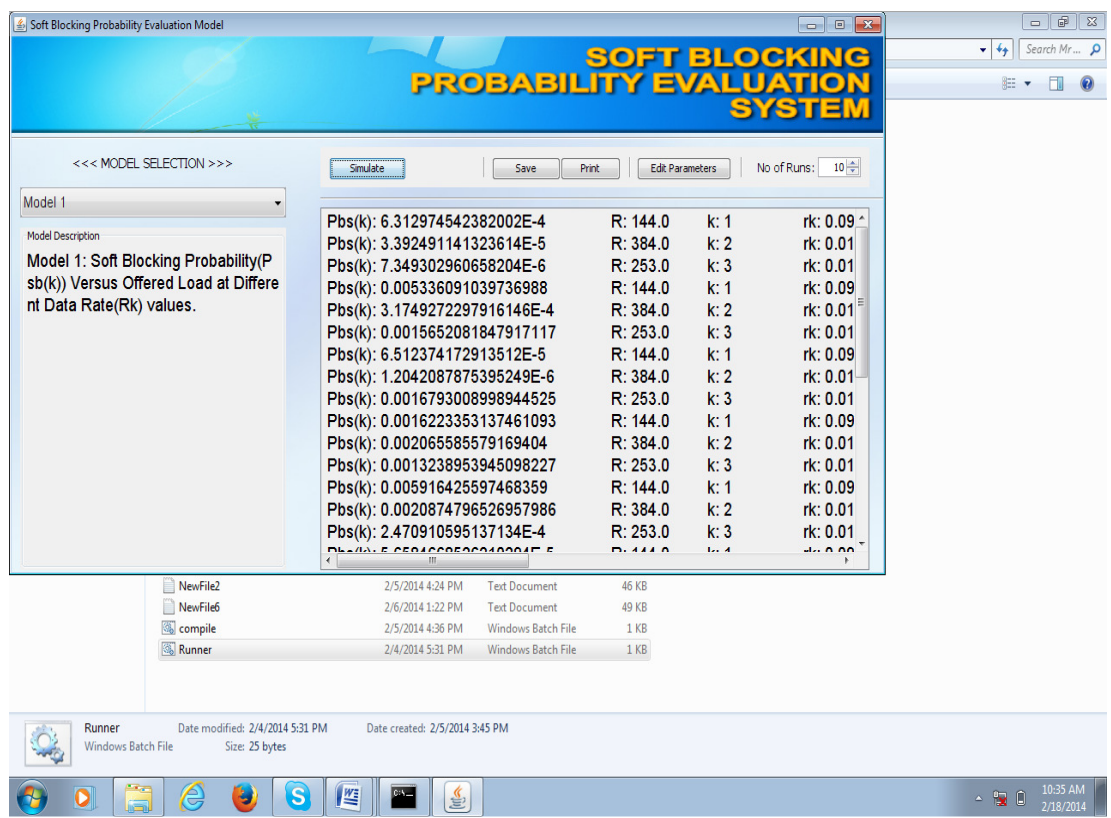

Figure 5: Soft blocking results for performance measure 1

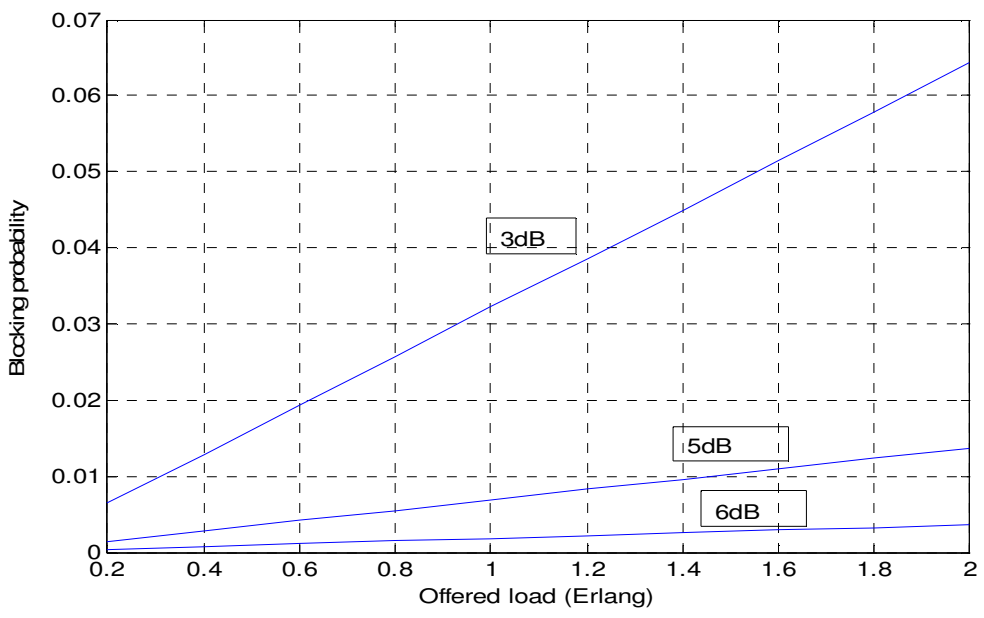

Figure 6a: Soft blocking results for performance measure 2 in MATLAB 
International Journal of Wireless \& Mobile Networks (IJWMN) Vol. 6, No. 4, August 2014

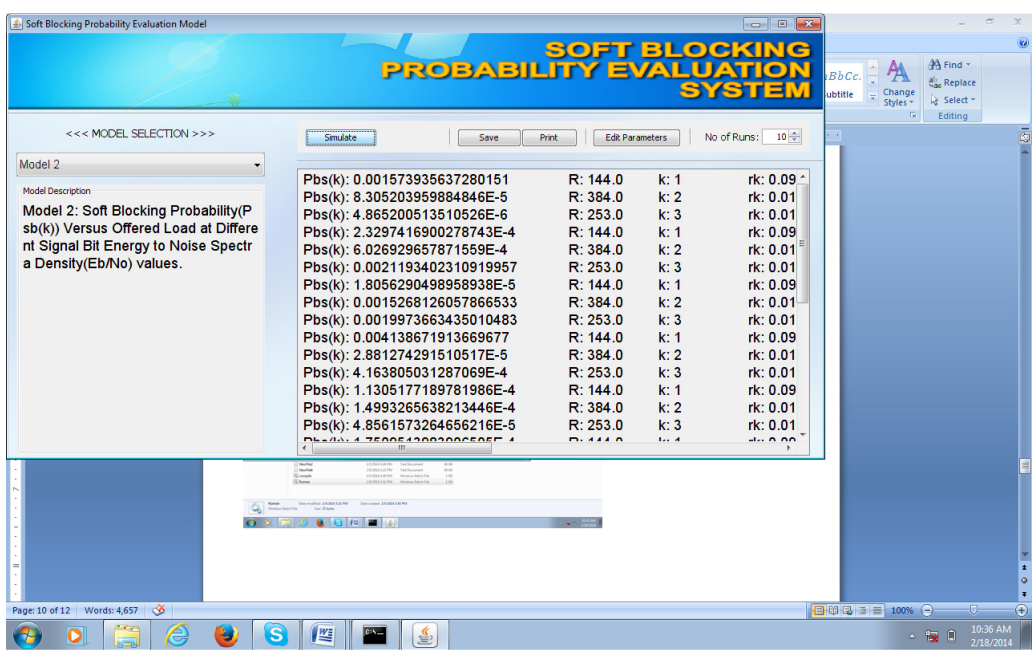

Figure 6b: Soft blocking results for performance measure 2 in JAVA

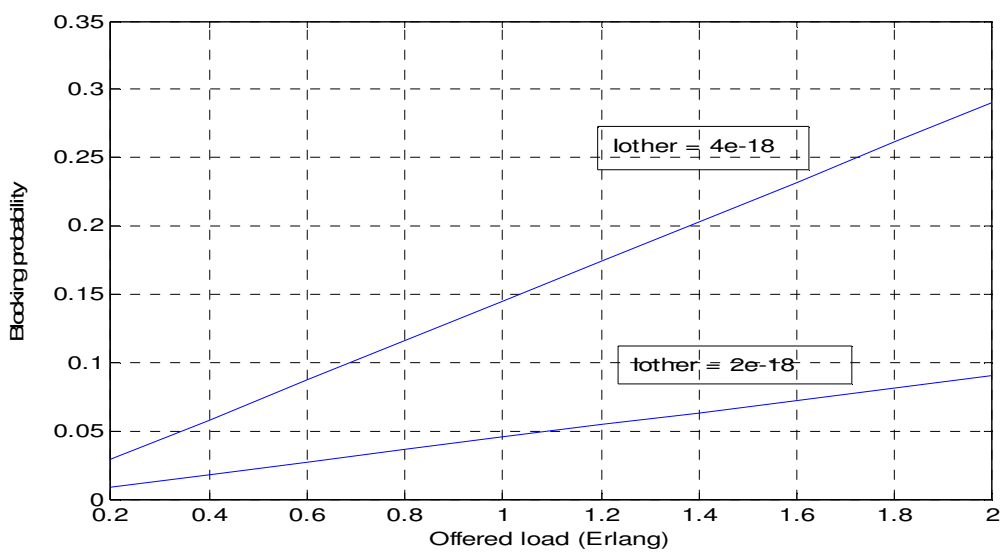

Figure 7a: Soft blocking results for performance measure 3 in MATLAB

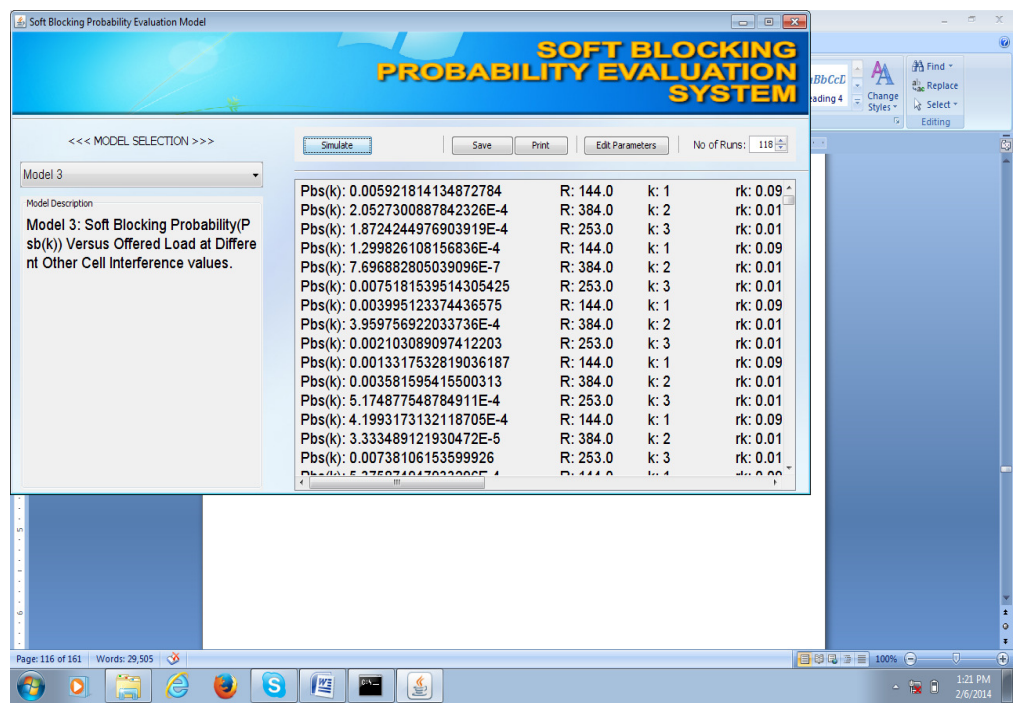

Figure 7b: Soft blocking results for performance measure 3 in JAVA 


\section{CONCLUSION}

We started from the verification that call blocking perceived negatively by users in mobile cellular networks actually exist at an alarming rate in a well established CDMA network Visafone in the region under study. As a solution, we developed a model for soft blocking probability evaluation by extending the Kaufman-Roberts algorithm which allows an efficient approximation of the blocking probabilities. We validate our results by simulation and show that the approximation yields accurate results even for large other-cell interferences and low user activities. Moreover, the impact of the model's parameters on soft blocking probability has been studied. This work showed that an efficient and fair resource management is possible for supporting traffic with strict QoS requirements. The results are particularly useful for operators who aim to predict the QoS of their networks for several combinations of the parameters (for dimensioning, prediction or optimization).

The imperfect power control framework considered addresses three main issues while ensuring that MSs achieve their QoS targets. First, it reduces the overall interference from neighboring cells, which is important for frequency reuse in multi-cell CDMA network. Second, it reduces the battery usage of MSs according to their individual preferences. Third, it mitigates the near-far problem by ensuring that MSs closest to the BS do not overpower the system at the detriment of those farther away.

\section{REFERENCES}

[1] Ramesh Babu H.S., Gowrishankar \& Satyanarayana P.S. (2009) "Call Admission Control Performance Model for Beyond 3G Wireless Networks”, (IJCSIS) International Journal of

Computer Science and Information Security, Vol. 6, No. 3, pp224-229.

[2] Karray, M. K. (2010) "Evaluation of the Blocking Probability and the Throughput in the Uplink of Wireless Cellular Networks", IEEE, In Proceedings of International Conference on

Communications and Networking (CommNet), Tozeur, Tunisia.

[3] Levine, D., Akyildiz, I. \& Naghshineh, M. (1997) “A Resource Estimation and Call Admission Algorithm for Wireless Multimedia Networks using the Shadow Cluster Concept", IEEE/ACM

Trans. Net., Vol. 5, No. 1, pp1-12.

[4] Epstein B. \& Schwartz, M. (2000) "Predictive QoS-based Admission Control for Multiclass Traffic in Cellular Wireless Networks”, IEEE JSAC, Vol. 18, No. 3, pp523-534.

[5] Choi, S. \& Shin, K. G. (2002) "Adaptive Bandwidth Reservation and Admission Control in QoSSensitive Cellular Networks”. IEEE Transactions on Parallel and Distributed Systems, Vol. 13.

[6] Chang, J., Chung, J. \& Sung, D. (2006) “Admission Control Schemes for Soft Handoff in DS-CDMA Cellular Systems Supporting Voice and Stream-type Data Services”, IEEE Trans.Vehic. Tech., Vol. 51, No.6, pp1445-14459.

[7] Yang, X. \& Bigham, J. (2007) “A Call Admission Control Scheme using NeuroEvolution Algorithm in Cellular Networks", IJCAI, pp186-191.

[8] Vassilakis, V. G. \& Logothetis, M. D. (2008) "The Wireless Engset Multi-Rate Loss Model for the Handoff Traffic Analysis in WCDMA Networks", IEEE.

[9] Parkinson, R. (2005) Traffic Engineering Techniques in Telecommunications, Infotel Systems Inc. Retrieved 2012-10-17 from http://www.tarrani.net/mike/docs/TrafficEngineering.pdf

[10] Zukerman, M. (2008) An Introduction to Queuing Theory and Stochastic Teletraffic Models Retrieved 2012-11-27 from http://www.ee.cityu.edu.hk/ zukerman/classnotes.pdf 
International Journal of Wireless \& Mobile Networks (IJWMN) Vol. 6, No. 4, August 2014

[11] Quo, J., Chan, S., Wong, E. W. M., Zukerman, M., Taylor, P. \& Tang, K. S. (2003) "On Blocking Probability Evaluation for Video-on-Demand Systems".

[12] Kaufman, J. (1981) "Blocking in a Shared Resource Environment”, IEEE Trans.Commun., Vol. 29, No. 10, pp1474-1481.

[13] Roberts, J. W. (1981) “A Service System with Heterogeneous User Environments”, in G. Pujolle (Ed.), Performance of Data Communications Systems and their Applications, North-Holland, Amsterdam, pp423-431.

[14] Andrew, L. L. H., Payne, D. J. B. \& Hanly, S. V. (1999) "Queuing Model for Soft-blocking CDMA Systems", IEEE, pp436-440.

[15] Verdone, R., Orriss, J., Zanella, A. \& Barton, S. K. (2002) "Evaluation of the Blocking Probability in a Cellular Environment with Hard Capacity: A Statistical Approach", In Proceedings of 13th IEEE International Symposium on person, Indoor and Mobile Radio Communications, Vol. 2: pp658-622.

[16] Bonald, T. \& Proutiere, A. (2005) "Conservative Estimates of Blocking and Outage Probabilities in CDMA Networks”. Performance Evaluation, Vol. 62, No. 14, pp50-67.

[17] Razavilar, J. Farrokhi, F. R. \& Liu, K. J. R., (2002) "Blocking Probability of Handoff Calls and Carried Traffic in Wireless Networks with Antenna Arrays", In Proceedings of 1st Asihomer Conference on Signals, Systems and Computers, Vol. 1, pp635-639.

[18] Huang, J., Huang, C. Y. \& Chou, C. M. (2004) "Soft-blocking Based Resource Dimensioning for CDMA Systems”, IEEE Veh. Tech. Conf. VTC2004, Vol.6, pp4306-4309.

[19] Kallos, G. A., Vassilakis, V. G. \& Logothetis, M. D. (2008) "Call blocking probabilities in a WCDMA Cell with Fixed Number of Channels and Finite Number of Traffic Sources".

[20] Hou, I. \& Kumar, P. (2009) “Admission Control and Scheduling for QoS Guarantees for Variable BitRate Applications on Wireless Channels”, in Proc. of MobiHoc'09, pp175-184.

[21] 3GPP TR 25.942 v10.0.0 (2011). Radio Frequency (RF) System Scenarios.

[22] Asuquo, D. E., Williams, E. E., Nwachukwu, E. O. \& Inyang, U. G. (2013) “An Intelligent Call Admission Control Scheme for Quality of Service Provisioning in a Multi-traffic CDMA Network", International Journal of Scientific and Engineering Research, Vol. 4, No. 12, pp152-161.

\section{Authors}

Dr Edem Williams is an Associate Professor of Computer Science at University of Calabar, Nigeria. He is also a Visiting Professor at Computer Science Department, University of Port Harcourt, Nigeria. He holds a PhD in Computer Science from University of Lagos, Nigeria, MSc in Computer Science from Manchester University and BSc in Computer Science from Obafemi Awolowo University, Ile-Ife, Nigeria. He is a member of IEEE, NCS and CPN. His research interest includes Artificial Intelligence, Database Management, Data Communications and Networks.

Daniel Asuquo received BSc in Computer Science from University of Calabar, Nigeria in 2002 and MSc in Computer Science from University of Ibadan, Nigeria in 2007. He is a lecturer in the department of Computer Science, University of Uyo, Nigeria. His research interest includes Wireless Communications and Mobile Networks, Web programming, Software Engineering, and Object-Oriented Programming. He is a member of Computer Professionals and Registration Council of Nigeria (CPN) and an associate member of Nigeria Computer Society (NCS).
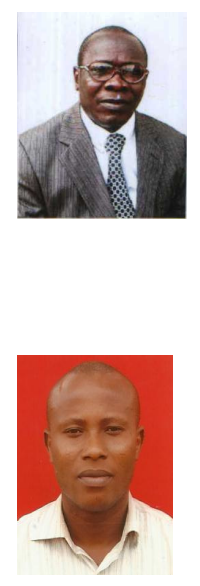